\title{
Trial Element Duration
}

National Cancer Institute

\section{Source}

National Cancer Institute. Trial Element Duration. NCI Thesaurus. Code C83440.

The period of time from start to finish of a trial element. 